\title{
A Comparison of Osteoblast Cell Proliferation and Osteocalcin Expression in Cuttlefish Bone and Bovine Bone Xenograft
} \author{
Oen Sindrawati ${ }^{3}$ \\ ${ }^{1}$ Dr. Soetomo General Hospital, Surabaya, Indonesia \\ ${ }^{2}$ Faculty of Medicine, Universitas Airlangga, Surabaya, Indonesia \\ ${ }^{3}$ Faculty of Medicine, Universitas Katolik Widya Mandala, Surabaya, Indonesia
}

Komang Agung Irianto ${ }^{1,2}$, Ameria Pribadi ${ }^{1,2}$, Ilham Abdullah Irsyam ${ }^{1,2}$, Yudhistira Pradnyan Kloping ${ }^{2}$,

Background: Cuttlefish bone Xenograft, calcium phosphate (CaP)-based biomaterial graft, offers an alternative and has been accepted for osteoconductive and probable osteo-inductive attributes. This study aims to compare the bone healing potential between the bovine-derived (BHA) and cuttlefish bone-derived (CHA).

Materials and Methods: The study compared osteoblast cell proliferation of $27 \mathrm{New}$ Zealand rabbits in $2.5 \mathrm{~mm}$ bone defect made in the femoral bone. The samples were divided into three groups, which were control, BHA and CHA group. The chemical and physical characteristics of BHA and CHA were determined for the content of hydroxyapatite by FourierTransform Infrared Spectroscopy (FTIR) and X-Ray Diffraction (XRD), then tested by Scanning Electron Microscopy (SEM) to evaluate the porosity. In the end of the second week, histopathologic and immunohistochemistry examinations were performed to evaluate the amount of osteoblast and osteocalcin expression.

Results: The FTIR, XRD and SEM analysis showed both BHA and CHA samples were hydroxyapatite according to Joint Committee on Powder Diffraction Standards (JCPDS). The CHA was significantly higher (297.22 \pm 19.772$)$ compared to BHA (258.22 \pm 30.926$)$ and control $(131.67 \pm 34.213)$. Osteocalcin expression in CHA $(7.82 \pm 2.230)$ compared to BHA $(6.09 \pm 3.724)$ and control (4.07 \pm 3.606$)$, was not significant $(p>0.05)$.

Conclusion: CHA group has the highest osteoblast cell proliferation and osteocalcin expression, meaning has a good potential as future source of bone graft.

Keywords: cuttlefish bone, bovine, bone graft, osteoblast cell

\section{Introduction}

Bone graft is a common technique in a bone defect to achieve secondary fracture healing where bone modelling and remodeling proceed. ${ }^{1}$ An ideal graft substitute should have bioresorbability, osteoconductive capacity, nontoxic, nonimmunogenic to the organism, easy to sterilize, and does not compromise mechanical stability ${ }^{2,3}$ Currently, autograft is the gold standard for bone graft because of its osteoconduction, osteoinduction, and osteogenesis ability., ${ }^{4,5}$

Date of submission: January 11, 2019

Last Revised: February 22, 2019

Accepted for publication: February 25, 2019

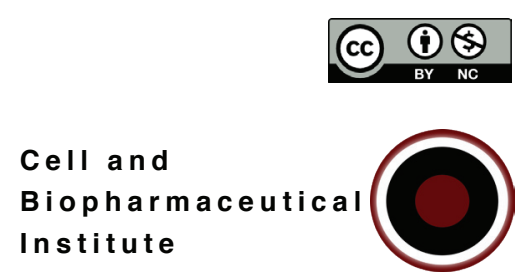

Corresponding Author:

Komang Agung Irianto Suryaningrat

Dr.Soetomo General Hospital/Universitas Airlangga

J. Prof. Dr. Moestopo 6-8, Surabaya 60115

E-mail: komang168@yahoo.com 
It is, however, associated with a high morbidity and limited availability $^{6,7}$, which pushes bone allografts to be used as natural substitute for bone defects ${ }^{1}$. Allograft also has a few disadvantages such as limited availability and risk of disease transmission, it presents some problems including rapid resorption, immunological rejection, infection, and technical difficulties in applying vascularized graft. ${ }^{5,6,8-11}$ Due to the limitations of autografts and allografts, xenografts are becoming a center of focus of studies to treat bone defects. ${ }^{12}$

Xenograft has been used frequently as bone graft substitutes with good results in recent years. Xenograft should contained hydroxyapatite biomaterial or calcium phosphate based that are similar to the human bone. ${ }^{5}$ Bovine bone shares the morphology, basic chemical composition, and crystallographic configuration of the mineral phase of the human bone, supporting new bone formation in contact area. ${ }^{7,8}$ It has been widely-used as a bone substitute because of its osteoconductive properties. It is also less susceptible to rejection or infection. ${ }^{13}$ In Indonesia, the most commonly used xenograft is bovine bone-derived. ${ }^{14}$ This material has been applied successfully in bone surgeries since they combine the morphology, basic chemical composition, and crystallographic configuration of the mineral phase of the bone. ${ }^{15-16}$ The use of bovine-derived xenograft (BHA) would utilize cow bones that are considered organic waste. These bovine bones have recently become a serious issue in coastal areas of Indonesia, hence a large-scale use of these otherwise useless product could contribute to a decrease in pollution in those areas. ${ }^{17}$ However, the data for clinical efficacy and success rate of it is limited. ${ }^{18}$

Another xenograft that is often used is a cuttlefish bone-derived xenograft (CHA). Cuttlefish bone is a natural biomaterial consisting mainly of calcium carbonate. ${ }^{3}$ It has an internal lamella matrix that is formed by an interconnected parallel sheet which creates a highly porous structure similar to coral. Previous studies have suggested the use of this material as a scaffold due to this property. ${ }^{10}$ It has an advantage compared to other alternatives as it contains aragonite that could convert into hydroxyapatite when processed hydrothermally. Because of its aragonite structure, it has been featured in several studies. ${ }^{16,19,20}$ It can also be obtained easily with a relatively cheap price globally. Due to its morphology and mineral structure, it can be shaped easily, hence making it compatibles with other types of bone structure with a high osteoinductive capacity. ${ }^{21,22}$

An ideal biomaterial for a scaffold should have the following characteristics: 1) High interconnected-porosity which supports cell growth and transport of nutrition and metabolism products; 2) Mechanical capability suitable to the implantation site; 3) Chemically appropriate surface area for adhesion, proliferation, and differentiation of cells with minimal rejection from the surrounding tissue; and 4) Biocompatibility and bioresorbability with a level of degradation and resorption time similar to a natural bone tissue. $^{23}$

Histologically, the fracture healing process incorporating osteoblast activation from resting state or osteogenesis from osteoprogenitor in periosteum and marrow cavity in the form of fibrocartilage callus formation. By the end of the second week, soft callus has built into hard callus as woven bone. Osteoblast cells quantity are used to examine osteoprogenitor cells differentiation capacity which indicates a progress towards physiological fracture healing. ${ }^{11}$ Osteocalcin is the most abundant non-collagenous protein in mature human bone, expressed during osteoblast differentiation. It is one of the reliable markers of bone formation. ${ }^{12}$

This study would like to compare the efficacy of bone fracture healing between BHA and CHA, by evaluating the amount of osteoblast cells (the nature of osteoconductive and osteo-inductive), and osteocalcin expression (osteoblast mature differentiation) in New Zealand white rabbit.

\section{Materials and methods}

This study was a comparative experimental study. The samples were 27 New Zealand white male rabbits each aged six to nine months, and weighed 2500 to 3000 grams. All the samples were in good health without any presence of diseases or abnormality.

The materials used were consist of a BHA processed in the Tissue Bank Laboratory of Dr. Soetomo General Hospital, Surabaya, and a CHA processed in hydrothermal reaction way in the Faculty of Science and Technology of Universitas Airlangga, Surabaya. The experimental unit used was freeze-dried hydroxyapatite xenograft pellet derived from each bone with gelatin (GEL) $10 \%$ as a binding material. To decrease the fragility of hydroxyapatite, surface modification by adding a binding material was done. ${ }^{24}$

A chemical characteristics analysis was performed in Clinical Pharmacy Laboratory of Universitas Airlangga, Surabaya, using Fourier Transform Infrared Spectroscopy (FTIR) to examine the Calcium $(\mathrm{Ca})$ and Phosphorus (P) content, X-Ray Diffraction (XRD) to 
examine the hydroxyapatite crystal content and Scanning Electron Microscope (SEM) to examine the porosity of each biomaterial. SEM showed pores visible with 200x magnification, the average macrospore's diameter of CHA $(104.46 \mu \mathrm{m})$ and BHA $(103.88 \mu \mathrm{m})$. The XRD analysis showed that the majority of samples consists of hydroxyapatite which fulfils the criteria of Joint Committee on Powder Diffraction Standards (JCPDS).

The characteristics analysis using FTIR showed an organic component with a functional group which corresponds to the wave's absorbance in a given frequency $\left(\mathrm{cm}^{-1}\right)$. The FTIR spectrum of BHA showed a peak wavelength of 3396.76 whereas the spectrum of CHA showsed a peak wavelength of 3311.89 and 3572.29. These indicated that $\mathrm{CHA}$ and $\mathrm{BHA}$ is a suitable hydroxyapatite based on the chemical structure of $\mathrm{Ca}_{10}\left(\mathrm{PO}_{4}\right)_{6}(\mathrm{OH})_{2}$, and were confirmed to be hydroxyapatite based on the criteria of JCPDS.

The study was conducted at the Institute of Tropical Disease and Laboratory of Anatomical Pathology, Faculty of Veterinary, Universitas Airlangga, Surabaya, and the ethical clearance was issued by the ethical research committee of the Faculty of Veterinary, Universitas Airlangga, Surabaya. The samples were adapted to their cages for a week before the study begins.

The rabbits were anesthetized intramuscularly on the left quadriceps muscle using atropine sulphate and diazepam. Oxytetracycline was given as a prophylactic antibiotic. Bone defects were made in each of the rabbit's distal metaphyseal region of the femur bilaterally with a 2.5 $\mathrm{mm}$ drill bit. The injured rabbits were then divided into the BHA group, the CHA group and the control group. The bone defect in the treatment group were implanted with bone grafts respectively according to the group. Wound care was performed on the third day and every five day afterwards.

On the end of the second week, the sample animals were sacrificed by resecting the distal femur where all samples were performed. The specimens were soaked in buffer formalin solution and slow decalcified afterwards using an acidic hydrochloric solution. The solution was changed daily until the specimens softened. A sagittal dissection was made in the affected bone and processed into paraffin block.

The hematoxylin and Eosin staining (HE) slides were further examined under the light microscope, osteoblast cells were counted for 10 fields of 40x magnification. Immunohistochemistry examination was also prepared to evaluate the osteocalcin expression. The statistical analysis (Kruskal-Wallis and Mann-Whitney test) were performed using SPSS version 21 (SPSS Inc., Chicago, USA).

\section{Results}

The osteoblast cell count measured from the HE-staining slides with a 40x magnification (Figure 1) showed that the CHA group have the highest number (297.22 \pm 19.772$)$ compared to the BHA group (258.22 \pm 30.926$)$ and control group (131.67 \pm 34.213$)$ (Table 1). According to the KruskalWallis test, the difference was statistically significant $(p<0.001)$.

Table 1. Osteoblast cell count comparison between groups.

\begin{tabular}{llccc}
\hline \multicolumn{1}{c}{ Group } & n & Mean & SD & $\boldsymbol{p}$-value \\
\hline Control & 9 & 131.67 & 34.213 & \\
BHA & 9 & 297.22 & 19.772 & $0.000^{*}$ \\
CHA & 9 & 258.22 & 30.926 & \\
\hline
\end{tabular}

Kruskal-Wallis test, $p<0.001$.

Osteocalcin expression of the osteoblast cells as dark brown cytoplasmic staining were highly expressed by the CHA group calculated according to the modified Remmele method (Figure 2). The CHA group expresses the highest amount of osteocalcin $(7.82 \pm 2.230)$ compared to the BHA group (6.09 \pm 3.724$)$ and control group (4.07 \pm 3.606$)$. However, the difference in osteocalcin expression was not significant (Table 2).

Table 2. Osteocalcin expression comparison between groups.

\begin{tabular}{llccc}
\hline \multicolumn{1}{c}{ Group } & $\mathbf{n}$ & Mean & SD & $\boldsymbol{p}$-value \\
\hline Control & 9 & 4.07 & 3.606 & \\
BHA & 9 & 6.09 & 3.724 & 0.114 \\
CHA & 9 & 7.82 & 2.230 & \\
\hline
\end{tabular}

Kruskal-Wallis test, $p<0.001$.

\section{Discussion}

BHA is commonly used in orthopedic and dental procedure and it is commercially available. It exhibits similar properties to human bone when processed in the right temperature. ${ }^{13}$ It has $75-80 \%$ porosity and a large 
$\mathbf{A}$

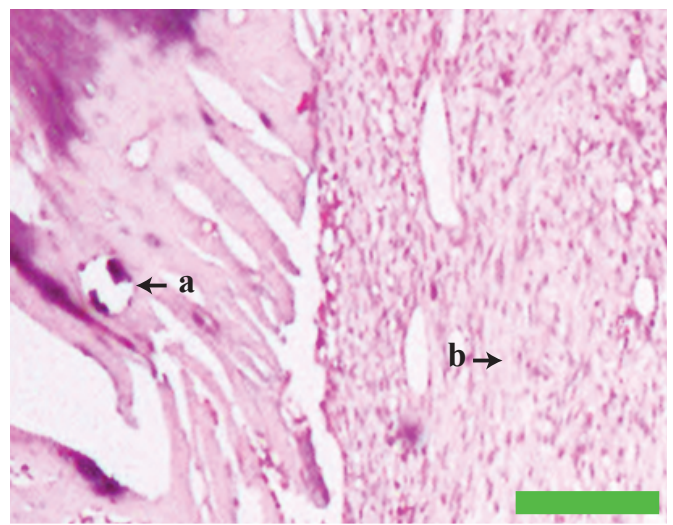

B

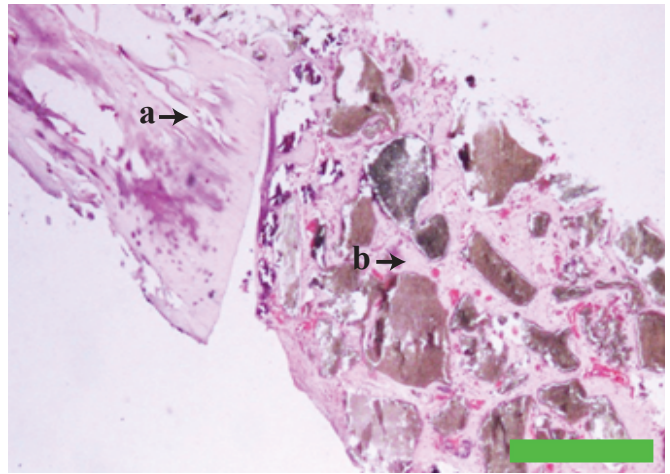

C

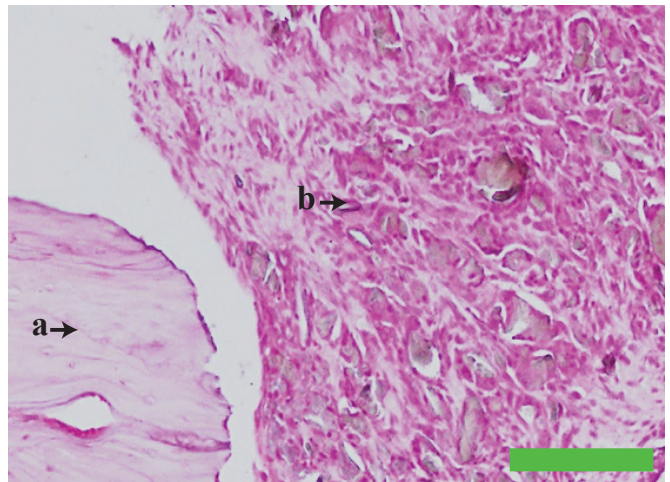

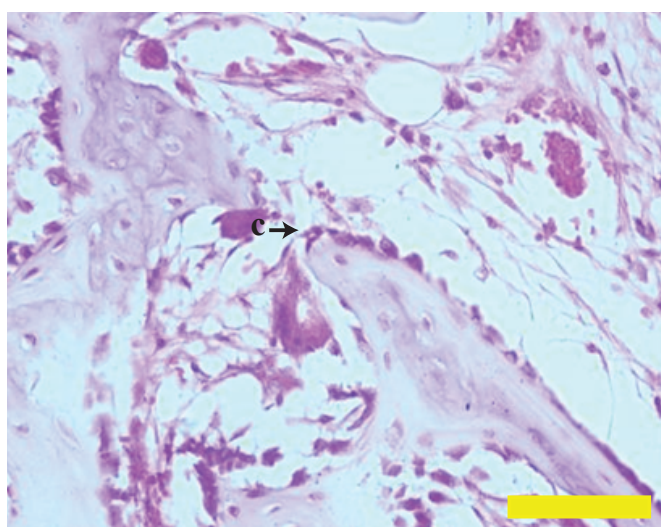
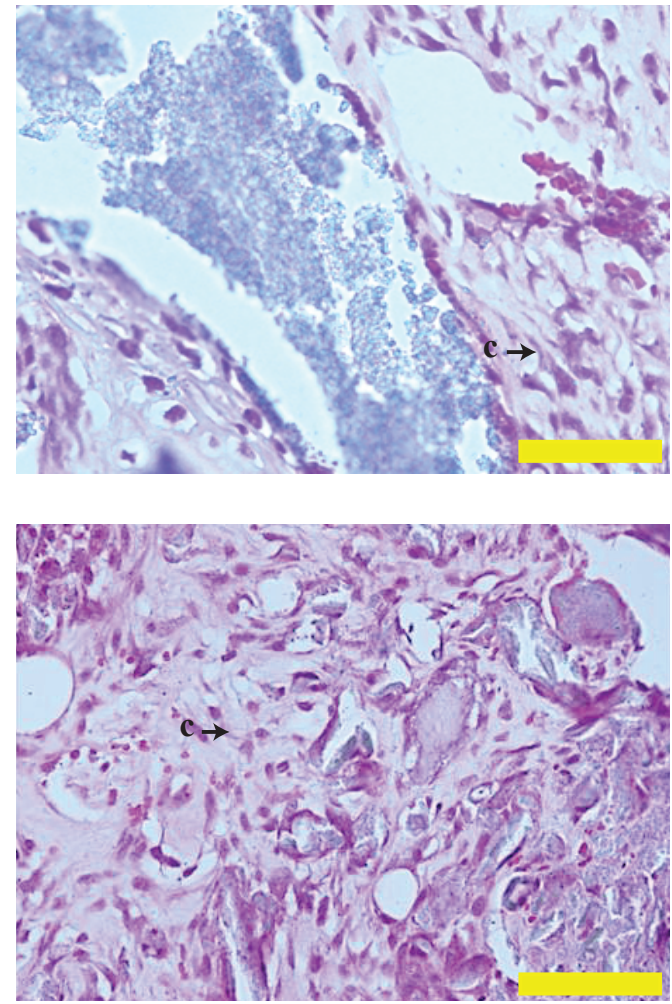

Figure 1. The histologic picture of the control (A), BHA (B) and CHA (C) group. a: cortical bone; b: fibrosis; c: osteoblastic cells; Green arrow: $10 \mu \mathrm{m}$ (H\&E; 10x); Yellow arrow: $2.5 \mu \mathrm{m}$ (H\&E; 40x).

interconnecting macropore and micropore system that facilitates angiogenesis and osteoblasts migration. ${ }^{14}$

The primary constituent of cuttlefish bone is calcium carbonate. ${ }^{16}$ The unique property of cuttlefish bone which has similar composition to human bones, is its high porosity which gives the cuttlefish its ability to float. Our study showed that CHA yields better osteoblast cell proliferation and higher osteocalcin production, therefore its structure is ideal as a scaffold. This is in accord with study reported by previous study which demonstrated that cuttlefish bone had excellent biocompatibility and osteogenic cell proliferation. ${ }^{17}$
The CHA is an excellent scaffold for the attachment of osteoblast activated from the resting lined in bone trabeculae and pro-osteoblast derived from progenitor in marrow. Dogan and Okumus reported cuttlefish bone graft had faster fibrous union formation during the initial week after implantation and showed better angiogenesis in the first week and by the end of observation. ${ }^{6}$ The viability of the cells significantly increased in less than a week after implantation. ${ }^{18}$

The concept of osteoconduction of hydroxyapatite is well accepted while the ability for osteoinduction not to mention osteogenesis are open for discussion and further 
A

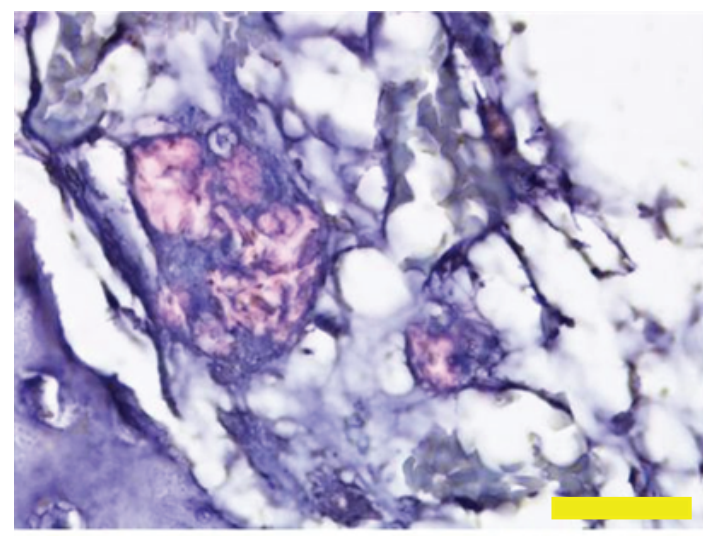

B

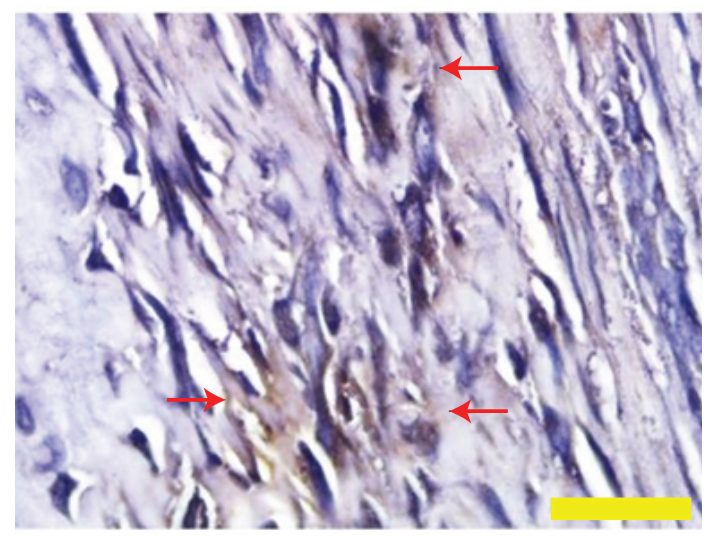

C

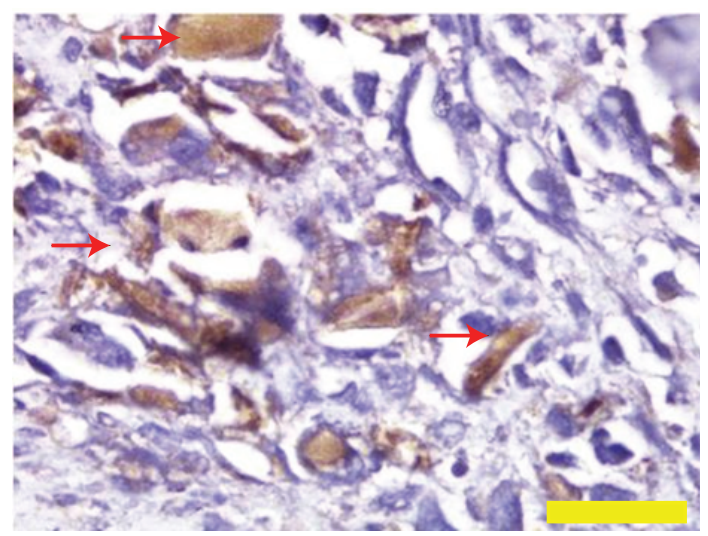

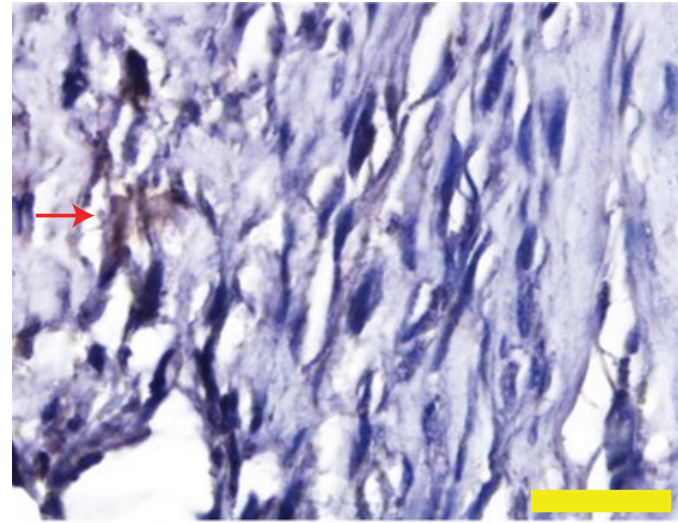
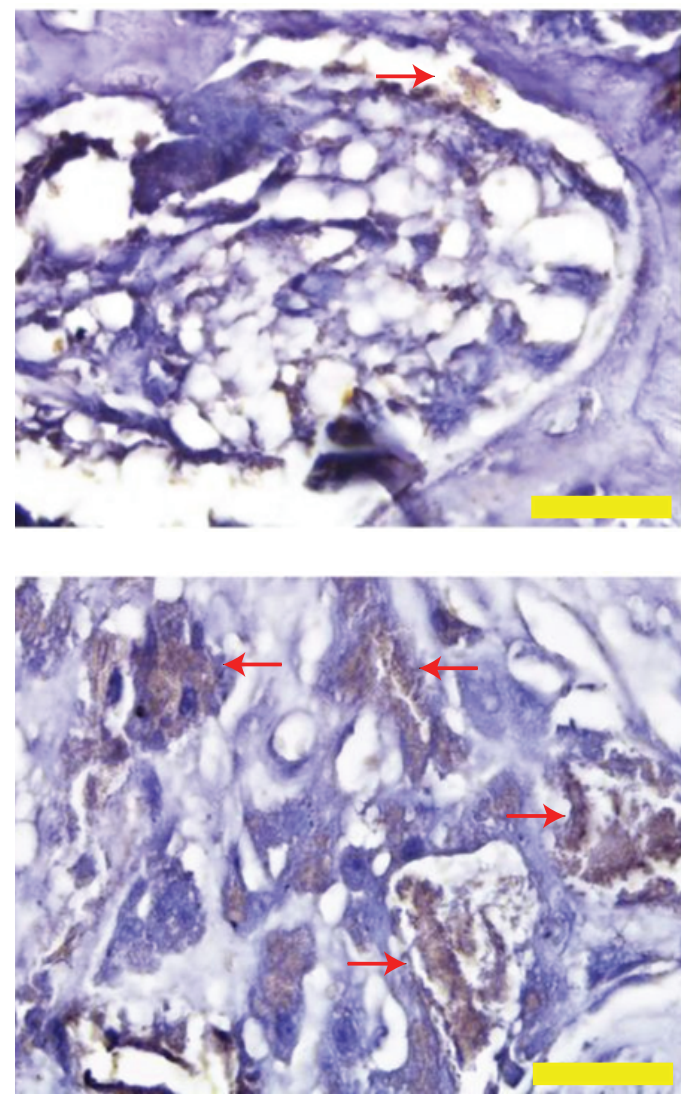

Figure 2. The Immunohistochemistry staining osteocalcin expression in control group (A), BHA (B) and CHA (C) group. Red arrow: dark brown cytoplasmic staining; Yellow bar: $2.5 \mu \mathrm{m}$.

research. A study found that cuttlefish bone contains other trace metals beside calcium, including magnesium. ${ }^{20}$ Magnesium ions are especially important in enhancing osteoblast cell attachment to orthopedic implant. ${ }^{21,22}$ Furthermore, magnesium has the ability to promote the proliferation and differentiation of osteoblast. ${ }^{23}$

In contrast to the significant higher osteoblast proliferation in CHA group, the osteocalcin expression which indicates osteoblasts maturation was not significantly different, although still higher compared to others. The osteocalcin expression naturally showed in hard callus as it is maturing. Radiological evaluation of CHA in 3 weeks implantation showed $100 \%$ hard callus formation compare to $75 \%$ callus in BHA. ${ }^{6}$

The result in this study suggested that CHA had compatible if not superior bone regenerative capability compared to BHA. Hopefully, this material could be applied clinically in the future as it offers a cheap, abundant alternative. ${ }^{24,25}$ 
The authors also realized the limitations of this study. The observation made in this study was one time only, at the end of second week after implantation. Further studies should incorporate continuous observation in each following week to better observe the degree of osteoblast maturation and osteocalcin expression in different stages of healing and proceed to evaluate the remodeling process which implicate the whole fracture healing. The authors also hope with this successful animal study, future clinical human application is achievable in near future.

\section{Conclusion}

Hydroxyapatite of the CHA yielded a higher osteoblast cell number compared to the BHA and control group. Furthermore, cuttlefish bone also presents a higher expression of osteocalcin which indicates mature osteoblast albeit insignificant statistically.

\section{Acknowledgements}

This study was supported by the grant from the Clinical Research Unit of Dr. Soetomo General Hospital, Surabaya.

\section{References}

1. Wang W, Yeung KWK. Bone grafts and biomaterials substitutes for bone defect repair: a review. Bioact Mater. 2017; 2(4): 224-47.

2. Janicki P, Schmidmaier G. What should be the characteristics of the ideal bone graft substitute? Combining scaffolds with growth factors and/or stem cells. Injury. 2011; 42(Suppl 2): S77-81.

3. Kim BS, Kang HJ, Yang SS, Lee J. Comparison of in vitro and in vivo bioactivity: cuttlefish-bone-derived hydroxyapatite and synthetic hydroxyapatite granules as a bone graft substitute. Biomed Mater. 2014; 9(2): 25004. doi: 10.1088/1748-6041/9/2/025004.

4. Chaves MD, de Souza Nunes LS, de Oliveira RV, Holgado LA, Filho $\mathrm{HN}$, Matsumoto MA, et al. Bovine hydroxyapatite (Bio-Oss((R))) induces osteocalcin, RANK-L and osteoprotegerin expression in sinus lift of rabbits. J Craniomaxillofac Surg. 2012; 40(8): e315-20.

5. Bahrololoom ME, Javidi M, Javadpour S, Ma J. Characterisation of natural hydroxyapatite extracted from bovine cortical bone ash. J Ceram Process Res. 2009; 10(2): 129-38.

6. Dogan E, Okumus Z. Cuttlebone used as a bone xenograft in bone healing. Vetmed. 2014; 59(5): 254-60.

7. Battistella E, Mele S, Foltran I, Lesci IG, Roveri N, Sabatino P, et al. Cuttlefish bone scaffold for tissue engineering: a novel hydrothermal transformation, chemical-physical, and biological characterization. J Appl Biomater Funct Mater. 2012; 10(2): 99-106.

8. Rocha JHG, Lemos AF, Agathopoulos S, Kannan S, Valério P, Ferreira JM. Hydrothermal growth of hydroxyapatite scaffolds from aragonitic cuttlefish bones. J Biomed Mater Res A. 2006; 77(1): $160-8$.
9. Ledford CK, Nunley JA 2nd, Viens NA, Lark RK. Bovine xenograft failures in pediatric foot reconstructive surgery. J Pediatr Orthop. 2013; 33(4): 458-63.

10. Ivankovic H, Tkalcec E, Orlic S, Ferrer GG, Schauperl Z. Hydroxyapatite formation from cuttlefish bones: kinetics. J Mater Sci Mater Med. 2010; 21(10): 2711-22.

11. Ghiasi MS, Chen J, Vaziri A, Rodriguez EK, Nazarian A. Bone fracture healing in mechanobiological modeling: a review of principles and methods. Bone Reports. 2017; 6: 87-100.

12. Seibel MJ. Biochemical markers of bone turnover part I: biochemistry and variability. Clin Biochem Rev. 2005; 26(4): 97-122.

13. Ooi CY, Hamdi M, Ramesh S. Properties of hydroxyapatite produced by annealing of bovine bone. Ceram Int. 2007; 33(7): 1171-7.

14. Artzi Z, Weinreb M, Givol N, Rohrer MD, Nemcovsky CE, Prasad $\mathrm{HS}$, et al. Biomaterial resorption rate and healing site morphology of inorganic bovine bone and beta-tricalcium phosphate in the canine: a 24-month longitudinal histologic study and morphometric analysis. Int J Oral Maxillofac Implants. 2004; 19(3): 357-68.

15. Berberi A, Samarani A, Nader N, Noujeim Z, Dagher M, Kanj W, et $a l$. Physicochemical characteristics of bone substitutes used in oral surgery in comparison to autogenous bone. Biomed Res Int. 2014; 2014; 320790. doi: 10.1155/2014/320790.

16. Neto AS, Ferreira JMF. Synthetic and marine-derived porous scaffolds for bone tissue engineering. Materials (Basel). 2018; 11(9): E1702. doi: 10.3390/ma11091702.

17. Kim BS, Kim JS, Sung HM, You HK, Lee J. Cellular attachment and osteoblast differentiation of mesenchymal stem cells on natural cuttlefish bone. J Biomed Mater Res - Part A. 2012; 100A(7): 16739.

18. Lee H, Jang TS, Song J, Kim HE, Jung HD. The production of porous hydroxyapatite scaffolds with graded porosity by sequential freeze-casting. Materials (Basel). 2017; 10(4): E367. doi: 10.3390/ ma10040367.

19. Sotto-Maior BS, Senna PM, Aarestrup BJ V, Ribeiro RA, NM de Souza Picorelli Assis, Del Bel Cury AA. Effect of bovine hydroxyapatite on early stages of bone formation. Rev Odonto Cienc. 2011; 26(3): 198-292.

20. Yildiri, OS, Okumus Z, Kizilkaya M. Comparative quantative analysis of sodium, magnesium, potassium and calcium in healthy cuttlefish backbone and non-pathological human elbow bone. Can J Anal Sci Spectrosc. 2007; 52(5): 270-5.

21. Park JW, Kim YJ, Jang JH, Song H. Osteoblast response to magnesium ion-incorporated nanoporous titanium oxide surfaces. Clin Oral Implants Res. 2010; 21(11): 1278-87.

22. Zreiqat H, Howlett CR, Zannettino A, Evans P, Schulze-Tanzil G, Knabe $\mathrm{C}$, et al. Mechanisms of magnesium-stimulated adhesion of osteoblastic cells to commonly used orthopaedic implants. J Biomed Mater Res. 2002; 62(2): 175-84.

23. Mourin V, Cattalini JP, Boccaccini AR. Metallic ions as therapeutic agents in tissue engineering scaffolds: an overview of their biological applications and strategies for new developments. J R Soc Interface. 2012; 9(68): 401-19.

24. Okumufi $Z$. The cuttlefish backbone: a new bone xenograft material? Turk J Vet Anim Sci. 2005; 29: 1177-84.

25. Kloping LP, Purwati P, Edward M. The healing effect of cuttlefish bone on fractured bone in rat model. Bali Med J. 2016; 5(2): 193196. 